\title{
CC AND CXC CHEMOKINES PATTERNS IN PSORIASIS DETERMINED BY PROTEIN ARRAY METHOD WERE INFLUENCED BY GOECKERMAN'S THERAPY
}

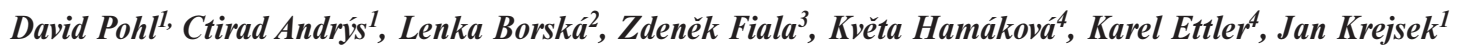

Charles University in Prague, Faculty of Medicine and University Hospital Hradec Králové, Czech Republic: Department of Clinical Immunology and Allergy ${ }^{1}$; Department of Pathological Physiology2; Department of Hygiene and Preventive Medicine $^{3}$; Department of Dermatology and Venereology 4

\begin{abstract}
Summary: Goeckerman's therapy (GT) of psoriasis is based on daily application of pharmacy grade coal tar on affected skin with subsequent exposure to UV light. The aim of this study was to evaluate the influence of Goeckerman's therapy of psoriasis on the levels of proangiogenic chemokines ENA-78 (CXCL5, Epithelial Cell Derived Neutrophil Attractant78), GRO alpha (CXCL1, Growth-Related Oncogene), IL-8 (CXCL8, Interleukin-8), MCP-1 (CCL2, Monocyte Chemotactic (Chemoattractant) Protein 1) and RANTES (CCL5, Regulated on Activation of Normal T Cell Expressed and Secreted) in peripheral blood of 22 children's patients with psoriasis. 22 otherwise healthy children serve as a control group. The serum levels of chemokines were determined by commercial membrane protein array technique (RayBiotech, USA). Efficacy of Goeckerman's therapy was delineated by PASI score. Disease activity was significantly diminished by Goeckerman's therapy ( $p<0.001$ ). Serum levels of GRO alpha and MCP-1 in patients before GT were significantly higher than those measured in healthy blood donors (GRO alpha: $p=0.0128$ and MCP-1: $p=0.0003$ ). Serum levels of GRO alpha, MCP-1 and RANTES were significantly diminished by GT (GRO alpha: $p=0.002$, MCP-1: $p=0.048$ and RANTES: $\mathrm{p}=0.0131$ ). Compared to the healthy controls, serum level of MCP-1 remained significantly increased in psoriasis patients after GT $(\mathrm{p}<0.0001)$. In conclusion, we found that the GT of psoriasis influenced the serum levels of proinflammatory and proangiogenic chemokines, especially GRO alpha, MCP-1 and RANTES. It could be the cause for decreased proangiogenic activity which is described after GT of psoriasis.
\end{abstract}

Key words: Psoriasis; Goeckerman's therapy; Angiogenesis; Chemokines; Protein array

\section{Introduction}

The original Goeckerman's method of therapy (GT) of psoriasis, which is based on daily application of pharmacy grade coal tar on affected skin with subsequent exposure of the body surface to UV radiation, was introduced by William Goeckerman in 1925 (14). In spite of the potentially increased genotoxic risk due to direct exposure of the skin to the poorly defined hydrocarbons present in a coal tar, this therapy is preferred for several reasons. Amongst them, easy administration, low cost and good clinical response followed by long-term remission are the most important $(20,21)$. Psoriasis is now recognized as a T-cell driven immune-mediated systemic inflammatory disease with skin manifestation characterized by hyperproliferation and abnormal differentiation of keratinocytes, combined with abundant endothelial cell proliferation and deregulation of cell apoptosis $(15,25)$. The typical feature of psoriasis is an epidermal hyperproliferation which is closely linked to the high angiogenic activity. This angiogenic activity is firmly established in psoriasis $(8,22)$. Highly intricate process of angiogenesis is carefully regulated by numerous intimate cell-to-cell contacts and humoral interactions involving cytokines, growth and differentiation factors, matrix metalloproteinases, tissue inhibitors of matrix metalloproteinases and chemokines $(7,8)$.

Chemokines are characterized by four conserved cysteine residues and nearly all of these cytokines have now been shown to chemoattract and activate different leukocyte subsets. The chemokine family of cytokines can be divided into subfamilies based on the positioning of the cysteine residues. In the chemokine "CXC" subfamily, the first two cysteine residues are separated by an intervening variable amino acid. In the chemokine "CC" subfamily, the first two cysteine residues are adjacent (1).

Angiogenesis is an integral part of immunopathogenesis of many other chronic inflammatory diseases such as rheumatoid arthritis, psoriasis and retinopathy $(10,22)$. 
The growth and penetration of new blood vessels into sites of inflammation has a vital role in this process and can be directly affected by chemokines. The structural amino acid motif known as ELR (Glu-Leu-Arg) within the chemokine molecules which is present in some chemokines determines their capacity to promote angiogenesis $(1,29)$.

Process of angiogenesis is based on the mutual interplay between endothelial cells and fibroblasts. The network of extracellular matrix molecules, including collagen fibrils, fibronectin and vitronectin, is essential for oriented endothelial cell migration which is driven by chemokines released from various proteoglycanes moieties during inflammatory response. Without the presence of extracellular matrix fibrils, there is no formation of new blood vessels in tissue. It is firmly proven by numerous studies that fibroblasts are the richest source of extracellular matrix molecules in the body. The CXC chemokines IL-8 and GRO-alpha regulating fibroblast activation and proliferation are thus of immense importance in the angiogenesis $(1,19)$.

Abnormalities in angiogenesis play the critical role in the inflammatory response in psoriasis $(8,22)$. Blood vessel neoformation is an integral part of the pathogenesis of many immunopathological diseases. Both keratinocytes and infiltrating inflammatory immune cells are rich sources of cytokines, chemokines, growth factors and mediators with proangiogenic activity in patients with psoriasis. The list of such biologically active compounds is impressive and includes, amongst others, pluripotent proinflammatory cytokines e.g. TNF $\alpha$; IL-8 and other chemokines, and growth factors VEGF and bFGF $(23,28,30)$.

The aim of this study was to follow the serum levels of proinflammatory and proangiogenic chemokines in patients with psoriasis treated by GT with the help of a newly developed technique of protein array. In the course of this study, elevated levels of proangiogenic chemokines in peripheral blood of patients with psoriasis were revealed. The inhibitory effect of Goeckerman's therapy on these proangiogenic chemokines was further delineated in this study.

\section{Material and Methods}

Altogether, 22 children's patients with chronic plaque form of psoriasis were enrolled to this study. The study was approved by the Ethics Committee of the University Hospital in Hradec Kralove. Informed written consent was obtained from parents of each patient and healthy controls. Our study group 22 children consisted of 15 girls and 7 boys (average age: 13.6 years, range: $8-18$ years). Twenty two otherwise healthy children, 13 girls and 9 boys (average age: 15.4 years, range: $7-17$ years) served as a control group. Control group was recruited by clinical dermatologist from the cohort of children referred to the clinical examinations with negative resolution.

Goeckerman's therapy was indicated by the consulting dermatologist, with patient-to-patient adjustments based on the activity of the disease. Average duration of therapy was 24 days (range: 12-30 days). Coal tar ointment with $5 \%$ pharmaceutical grade coal tar (Pix Lithanthracis) was applied daily on affected skin (10-75\% of total body surface). Patients were irradiated with UV radiation daily. Duration of UV irradiation was individual, depending on the disease activity (range 1-15 minutes). The light beam density (dose) was controlled by Sola - Scope 2000 spectrometer (Solatell, UK) and was $245.60 \mu \mathrm{W} / \mathrm{cm}^{2}$ for UV-B radiation and $134.4 \mu \mathrm{W} / \mathrm{cm}^{2}$ for UV-A radiation.

Previous exposure of patients to UV radiation and polyaromatic hydrocarbons was assessed by a questionnaire. Patients with positive personal history were excluded from the study. The efficacy of Goeckerman's therapy was assessed by clinical evaluation of erythema, desquamation, and skin infiltration using PASI score (9). GT is ceased when $80 \%$ decrease of PASI is achieved. A patient's Psoriasis Area and Severity Index (PASI) is a measure of overall psoriasis severity and coverage. In short, for the PASI score evaluation, the body is divided into four sections. Each of these areas is scored by itself, and then the four scores are combined into the final PASI. The four areas are: the legs, the body, the arms, and the head. The area of skin involved is measured for each skin section, and expressed as a percentage of the skin of a particular area. In addition, the severity is estimated by four different parameters: itching, erythema, scaling and thickness. For each skin section, up the four severity scores are added, than multiplied by the total area score. The result of this calculation is then multiplied by the percentage of skin in particular section. Finally, the PASI is the summa of these partial total skin section scores. PASI will range from 0 (healthy skin) to 96 (9). The PASI score was calculated before and after treatment for each patient.

Samples of venous blood were obtained by venipuncture of the forearm cubital vein before treatment and again after completion of Goeckerman's therapy at the day of dismissal from the hospital. Venous blood was also collected from otherwise healthy children who served as control. Peripheral blood samples were allowed to clot and serum was obtained after sample centrifugation. Serum samples were stored frozen at $-20^{\circ} \mathrm{C}$. Repeated thawing and freezing of samples were avoided.

Simultaneous analysis of selected chemokines was performed using antibody-based protein array method using RayBio Human Angiogenesis Antibody Array 1 manufactured by RayBiotech, Inc. USA, according to the instructions of manufacturer. Briefly, the method is based on the sandwich immunoassay principle. A panel of antibodies (capture) is immobilized in specific spot locations on the surface of membrane. Incubation of array membranes with biological samples results in the capture of cytokines by corresponding antibodies. The bound cytokines are detected with a cocktail of biotinylated antibodies. Signals are then visualized using streptavidin-HRP conjugate and colorimetry. Results are expressed in relative units as a color density of spots. 
Spot color density is reflecting concentration of the particular chemokine (Fig. 1). Color images of the membranes with spots are digitalized and assessed with the help of the special software ARES (ARay Evaluation System, Baria, Czech Republic).

Statistical analysis of data was performed using Med-

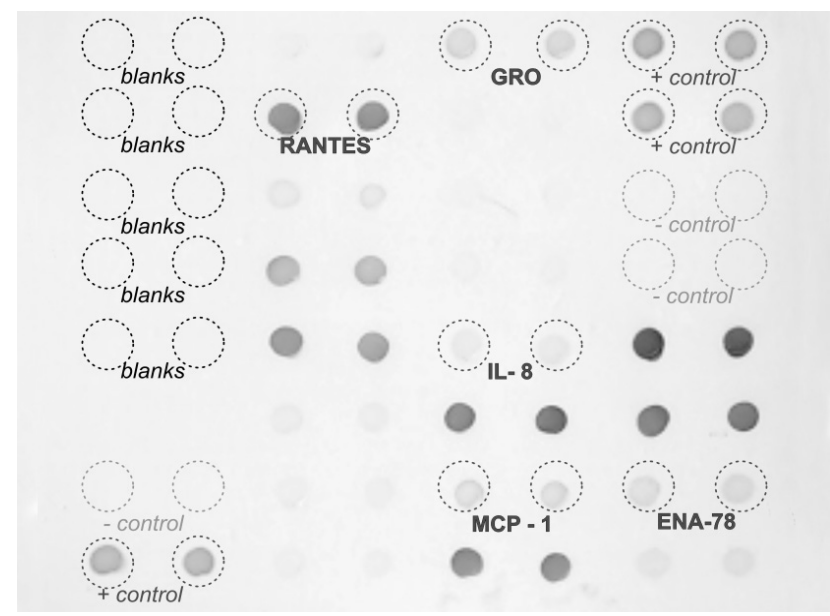

Fig. 1: A typical pattern of membrane protein array method for detection of angiogenic factors (spots relevant to this study are in a circle).

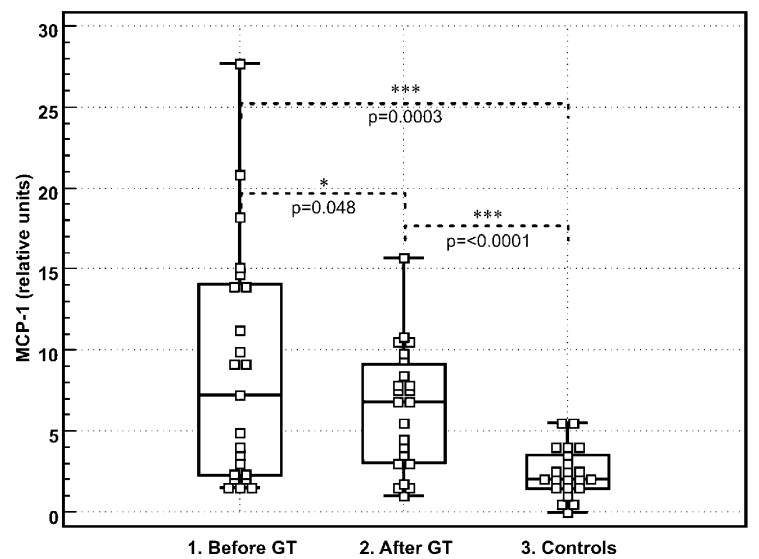

Fig. 3: The differences in the serum levels of MCP-1 (relative units) in patients with psoriasis before and after GT and comparison with healthy controls. A standard box-andwhisker plots (median, quartiles and range) were used.
Calc (MedCalc Software, Belgium) statistical software. Normal distribution of data was tested. In case of normal distribution groups of patients and controls were compared using Student's t-test. In other cases Wilcoxon's non-parametric test was used. Probability level less than 0.05 was considered as significant.

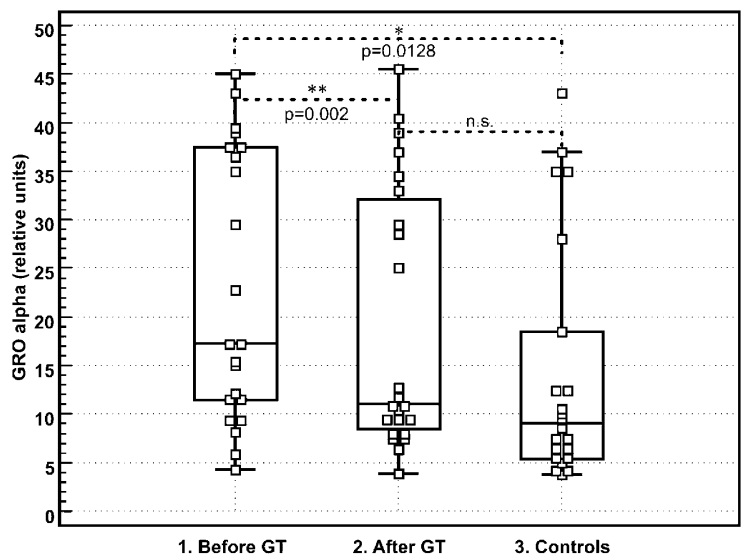

Fig. 2: The differences in the serum levels of GRO alpha (relative units) in patients with psoriasis before and after GT and comparison with healthy controls. A standard boxand-whisker plots (median, quartiles and range) were used.

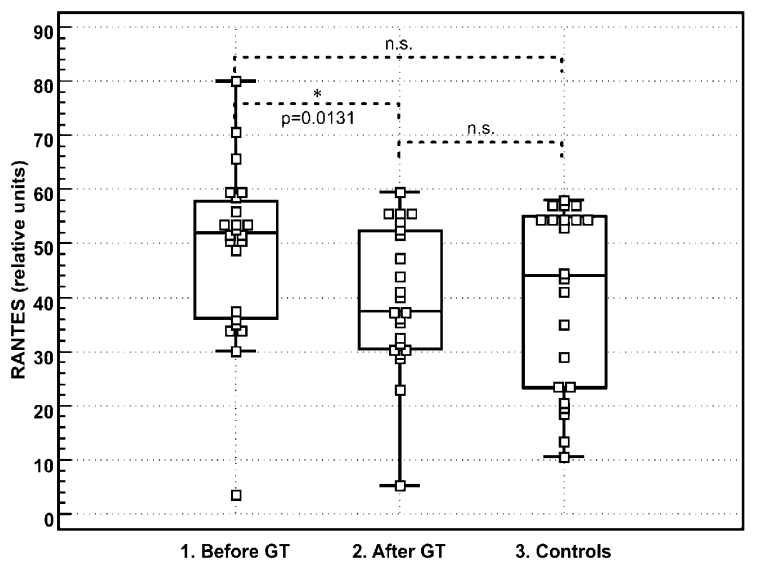

Fig. 4: The differences in the serum levels of RANTES (relative units) in patients with psoriasis before and after GT and comparison with healthy controls. A standard box-andwhisker plots (median, quartiles and range) were used.

Tab. 1: Descriptive statistic of acquired data (relative units).

\begin{tabular}{|l|c|c|c|c|c|c|c|c|c|}
\hline chemokine & \multicolumn{3}{|c|}{ before therapy } & \multicolumn{3}{c|}{ after therapy } & \multicolumn{3}{c|}{ Control group } \\
\hline & average & SD & median & average & SD & median & average & SD & median \\
\hline ENA-78 & 11.33 & 9.39 & 8.5 & 12.75 & 11.62 & 7.2 & 12.44 & 7.02 & 11.25 \\
\hline GRO $\alpha$ & 22.85 & 13.79 & 17.25 & 19.10 & 13.5 & 11.0 & 14.38 & 12.46 & 9.0 \\
\hline IL-8 & 14.17 & 19.73 & 5.40 & 10.70 & 12.07 & 5.0 & 7.14 & 10.83 & 4.75 \\
\hline MCP-1 & 8.69 & 7.27 & 7.2 & 6.47 & 3.77 & 6.80 & 2.43 & 1.49 & 2.0 \\
\hline RANTES & 49.02 & 15.91 & 52.0 & 39.76 & 13.07 & 37.5 & 39.97 & 16.97 & 44.0 \\
\hline
\end{tabular}




\section{Results}

All results were expressed in Tab. 1 as a means, medians and standard deviations (SD). Disease severity decreased significantly by Goeckerman's therapy. Pre-therapy PASI scores $20.33 \pm 9.86$ dropped to $7.71 \pm 5.22$ after therapy $(p<0.001)$. No significant correlations between serum levels of any parameter tested and disease activity expressed as PASI score were found.

In comparison to the levels in healthy blood donors, serum levels of GRO alpha and MCP-1, but not RANTES, ENA-78 and IL-8 in patients before Goeckerman's therapy were significantly higher (Fig. 2, 3). Serum levels of MCP1, GRO alpha, and RANTES were significantly diminished after Goeckerman's therapy (Fig. 2, 3, 4).

Compared to the healthy controls, serum levels of MCP-1 remained significantly increased in psoriasis patients after GT (Fig. 3).

\section{Discussion}

It is well established that Goeckerman's therapy of psoriasis (GT), which is based on the combination of antiinflammatory and immunomodulatory effects of poorly characterized aliphatic and aromatic hydrocarbons present in pharmaceutical grade coal tar and well documented immunosuppressive and immunomodulatory activities of UV irradiation, is highly efficient in the treatment of psoriasis. GT produces good clinical response and long term remission is achieved in a majority of patients. This complex therapeutical regimen has numerous targets in the pathophysiology of psoriasis. The abnormal hyperproliferative capacity of epidermal cells is known to be diminished by this therapy $(14,20)$. In contrast, little is known about the influence of Goeckerman's therapy of psoriasis on angiogenic activity which is abnormal in these patients $(3,5)$. Our study addressed whether Goeckerman's therapy influences the serum level of proangiogenic chemokines ENA-78, GRO-alpha, IL-8, MCP-1 and RANTES in children with psoriasis.

Increased production of chemokines RANTES, IL-8, MCP-1 and GRO alpha by activated keratinocytes was proven by numerous authors $(12,13,24,27)$. Chemokines secretion is induced by many stimuli. Amongst them, proinflammatory and immunomodulatory cytokines TNF alpha and interferon gamma are the principal. The chemokine expression is downmodulated by some drugs including cyclosporine A, corticosteroids, vitamin D3 analog tacalcitol, or anti-TNF alpha biological therapy $(2,6,11,18)$, all of which are used in the treatment and having therapeutical effect in psoriasis. The level of RANTES is negatively influenced by UV-B irradiation which is an integral part of GT (4).

The evidence supporting the substantial role of angiogenesis in the immunopathogenesis of psoriasis is so established that anti-angiogenic therapy of psoriasis has been proposed as area of interest for the near future therapy $(8,26)$.
Soluble mediators such as chemokines are usually determined by immunochemical methods; ELISA technique being the most frequent. The measurement of a large spectrum of chemokines by ELISA is laborious and rather expensive. The new technology, so called multiplex assay enables us to follow several (tens) of these parameters in a single procedure in just a tiny volume of the examined sample. The small amount of sample which is needed for analysis is desirable especially in children.

Two principal approaches are utilized in multiplex assays. The first one is using multicolor immunofluorescence particle probes detected by flow cytometry (Luminex technology).

Another approach is membrane based antibody array method. In short, monoclonal antibodies highly specific for the particular chemokines are immobilised as spots onto membrane. The area of the membrane is incubated with examined serum. After incubation immobilised chemokines are visualized by a cocktail of second (labelled) antibodies. The development of colored spots takes places. These spots are evaluated by densitometry. This method has the advantage of having the sensitivity and specifity of enzymelinked-immunosorbent assays, and high-throughput of microspot arrays $(16,17,31)$.

To summarize our experience, it could be concluded, that multiplex protein assay could serve for the screen of many parameters to identify those markers which seems to be promising for further analysis by techniques with higher levels of accuracy and reproducibility, such as ELISA technique. In addition, the range of commercially available ELISA kits and antibodies is much more broad compared to multiplex protein assays.

\section{Conclusions}

In conclusion, we screened for proangiogenic factors by a novel antibody-based protein microarray technique. We found that the serum levels of proangiogenic chemokines GRO alpha, MCP-1 and RANTES which are increased in patients with psoriasis are significantly decreased after Goeckerman's therapy.

\section{Acknowledgment}

This study was supported by Ministry of Health, Czech Republic project No. MZO 00179906.

\section{References}

1. Adams DH, Lloyd AR. Chemokines: leucocyte recruitment and activation cytokines. Lancet 1997;349:490-5.

2. Albanesi C, Pastore S, Fanales-Belasio E, Girolomoni G. Cetirizine and hydrocortisone differentially regulate ICAM-1 expression and chemokine release in cultured human keratinocytes. Clin Exp Allergy 1998;28:101-9.

3. Andrys C, Borska L, Pohl D, Fiala Z, Hamakova K, Krejsek J. Angiogenic activity in patients with psoriasis is significantly decreased by Goeckerman's therapy. Arch Dermatol Res 2007;298:479-83.

4. Arakawa S, Hatano Y, Katagiri K, Terashi H, Fujiwara S. Effect of ultraviolet B irradiation on the production of regulated upon activation normal T-cell expressed and secreted protein in cultured human epidermal keratinocytes. Arch Dermatol Res 2006;297: 377-80. 
5. Arbiser JL, Govindarajan B, Battle TE, et al. Carbazole is a naturally occurring inhibitor of angiogenesis and inflammation isolated from antipsoriatic coal tar. J Invest Dermatol 2006;126:1396-1402

6. Benoit S, Toksoy A, Brocker EB, Gillitzer R, Goebeler M. Treatment of recalcitrant psoriasis with infliximab: effective reduction of chemokine expression. $\mathrm{Br}$ J Dermatol 2004;150:1009-12.

7. Bhushan M, Young HS, Brenchley PEC, Griffiths CEM. Recent advances in cutaneous angiogenesis. Br J Dermatol 2002;147:418-25.

8. Creamer D, Sullivan D, Bicknell R, Barker J. Angiogenesis in psoriasis. Angiogenesis 2002;5: 231-6.

9. De Rie MA, Goedkoop AY, Bos JD. Overview of psoriasis. Dermatol The 2004:17:341-9.

10. Folkman J. Angiogenesis. Annu Rev Med 2006;57:1-18

11. Fukuoka M, Ogino Y, Sato H, et al. RANTES expression in psoriatic skin, and regulation of RANTES and IL-8 production in cultured epidermal keratinocytes by active vitamin D3 (tacalcitol). Br J Dermatol 1998;138:63-70.

12. Gillitzer R, Ritter U, Spandau U, Goebeler M, Brocker EB. Differential expression of GRO-alpha and IL-8 mRNA in psoriasis: a model for neutrophil migration and accumulation in vivo. J Invest Dermatol 1996;107:778-82.

13. Gillitzer R, Wolff K, Tong D, et al. MCP-1 mRNA expression in basal keratinocytes of psoriatic lesions. J Invest Dermatol 1993;101:127-31.

4. Goeckerman WH. Treatment of psoriasis. Northwest Med 1925;24:229-31.

15. Gudjonsson JE, Johnston A, Sigmundsdottir H, Valdimarsson H. Immunopathogenic mechanisms in psoriasis. Clin Exp Immunol 2004;135:1-8.

16. Huang RP. Detection of multiple proteins in an antibody-based protein microarray system. J Immunol Meth 2001;255:1-13.

17. Huang RP. Cytokine protein arrays. Meth Mol Biol 2004;264:215-31

18. Kojima T, Cromie MA, Fischer GJ, Voorhees JJ, Doder JT. GRO alpha mRNA is selectively overexpressed in psoriatic epidermis and is reduced by cyclosporine A in vivo, but not in cultured keratinocytes. J Invest Dermatol 19932:101:767-72.

19. Kulke R, Bornscheuer E, Schluter C, et al. The CXC receptor 2 is overexpressed in psoriatic epidermis. J Invest Dermatol 1998;110:90-4.
20. Lebwohl M, Ali S. Treatment of psoriasis. Part 1. Topical therapy and phototherapy. J Am Acad Dermatol 2001:45:487-98.

21. Lee E, Koo J. Modern modified 'ultra' Goeckerman therapy: a PASI assessment of a very effective therapy for psoriasis resistant to both prebiologic and biologic therapies. J DermatolTreat 2005;16:102-7.

22. Leong TT, Fearon U, Veale DJ. Angiogenesis in psoriasis and psoriatic arthritis: clues to disease pathogenesis. Curr Rheumatol Rep 2005;7:325-9.

23. Li J, Farthing PM, Thornhill MH. Oral and skin keratinocytes are stimulated to secrete monocyte chemoattractant protein-1 by tumour necrosis factor alpha and interferon gamma. J Oral Pathol Med 2000;29:438-44.

24. Li J, Ireland GW, Farthing PM, Thornhill MH. Epidermal and oral keratinocyte are induced to produce RANTES and IL- 8 by cytokine stimulation. J Invest Dermatol 1996;106: 661-6.

25. Lowes MA, Bowcock AM, Krueger JG. Pathogenesis and therapy of psoriasis Nature 2007;445:866-73.

26. Nielsen HJ, Christensen IJ, Svendsen MN, et al. Elevated plasma levels of vascular endothelial growth factor and plasminogen activator inhibitor-1 decrease during improvement of psoriasis. Inflamm Res 2002;51:563-7.

27. Raychaudhuri SP, Jiang WY, Farber EM, Schall TJ, Ruff MR, Pert CB Upregulation of RANTES in psoriatic keratinocytes: a possible pathogenic mechanism for psoriasis. Acta Derm Venereol 1999:79:9-11.

28. Steude J, Kulke R, Christophers E. Interleukin-1-stimulated secretion of interleukin- 8 and growth-related-oncogene-[alpha] demonstrates greatly enhanced keratinocyte growth in human raft cultured epidermis. J Invest Dermatol 2002; 119:1254-60.

29. Streiter RM, Polverini PJ, Kunkel SL, et al. The functional role of the ELR motif in CXC chemokine-mediated angiogenesis. J Biol Chem 1995;270:27348-57.

30. Tille JC, Wood J, Mandriota SJ, et al. Vascular endothelial growth factor (VEGF) receptor-2 antagonists inhibit VEGF - and basic fibroblast growth factor-induced angiogenesis in vivo and in vitro. J Pharmacol Exp Ther 2001:299:1073-85.

31. Wang CC, Huang RP, Sommer M, et al. Array-based multiplexed screening and quantitation of human cytokines and chemokines. J Prot Res 2002;1:337-43.

Submitted January 2009. Accepted April 2009.

\section{Corresponding author:}

RNDr. Ctirad Andrýs, Ph.D., University Hospital Hradec Králové, Department of Clinical Immunology and Allergy, Sokolská 581, 50005 Hradec Králové, Czech Republic; e-mail: andrys@lfhk.cuni.cz 